\title{
Analysis on Comprehensive Treatment of VOCs in Key Industries of A City
}

\author{
Zhipeng Zhuang ${ }^{1,2}$, Weijian Zhou ${ }^{1,2}$, Zhihua Pang ${ }^{2}$, Yutao Lei ${ }^{2}$, and Wenzhong Liang ${ }^{*}, 1,2$ \\ ${ }^{1}$ Guangzhou Huake Environmental Protection Engineering Co., Ltd., Guangzhou 510655, China \\ ${ }^{2}$ South China Institute of Environmental Sciences, Guangzhou 510655, China
}

\begin{abstract}
Vocs have become a weak base for air pollution control compared to particulate matter, sulfur dioxide and nitrogen oxide. Petrochemical, chemical, industrial coating, packaging and printing, oil storage and Marketing Industries (hereinafter referred to as key industries) are China's key sources of VOCs emissions. In order to win the battle of protecting the blue sky and improve the air quality, it is urgent to strengthen the comprehensive treatment of VOCs in key industries. In order to fully understand the comprehensive treatment and emission control of industrial sources of organic waste gases in the city, an updated survey was conducted on the emission of VOCs from industrial sources in the region, and the results of the survey were analyzed Analysis report on VOCs emission and comprehensive treatment in key industries.
\end{abstract}

\section{Pollution background and identification of key industries in a city}

\subsection{Background of VOCs pollution}

In China, the VOCs (volatile organic compounds) are organic compounds with saturated vapor pressure above 70.91 PA at room temperature and boiling point below $50{ }^{\circ} \mathrm{C}$ to $260{ }^{\circ} \mathrm{C}$ and initial distillation point equal to $250{ }^{\circ} \mathrm{C}$ at the atmosphere or any volatile organic solid or liquid at room temperature and pressure ${ }^{[1]}$. Vocs pollution emission has a prominent influence on atmosphere environment. Vocs are important precursors of fine particulate matter (PM2.5) and ozone (O3), and also contribute to climate change. In recent years, positive progress has been made in the control of PM2.5 pollution in China, especially in the beijing-tianjin-hebei region, the surrounding areas and the Yangtze River Delta Economic Zone, but the concentration of PM2.5 is still at a high level and the phenomenon of exceeding the standard is still widespread It is the key factor to win the battle of defending the blue sky and improve the air quality. The source apportionment of PM2.5 in beijing-tianjin-hebei region and its surrounding areas shows that organic matter $(\mathrm{Om})$ is the main component of PM2.5, accounting for $20 \%-40 \%$, and secondary organic matter accounts for $30 \%-50 \%$ of $\mathrm{OM}$, mainly from the conversion of VOCs. At the same time, the problem of $\mathrm{O} 3$ pollution is becoming more and more serious in our country. The study shows that VOCs are the main controlling factor of $\mathrm{O} 3$ generation in the key regions at present.

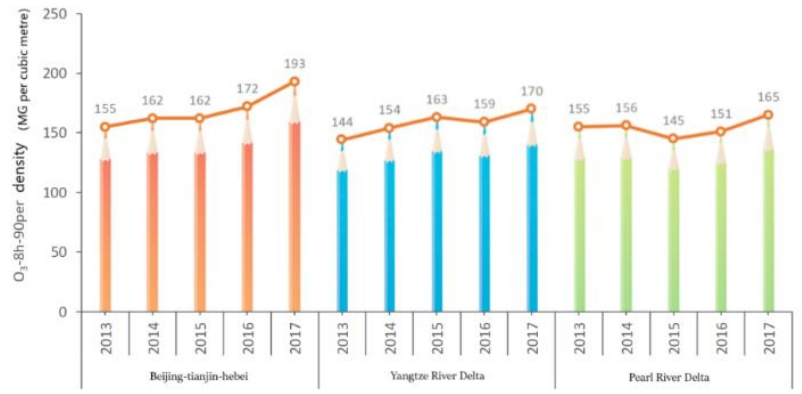

\subsection{Identification of key industries and major problems in a city}

Vocs-related industrial pollution enterprises in a city, mainly in the chemical industry, coating and printing industries. The main problems identified are as follows:

1.2.1. Activated Carbon adsorption method has low initial investment, but the actual operation cost is high, the management is difficult, the technical applicability is affected by many factors, it is difficult to reach the environmental protection standard stably.

1.2.2. The problem in the treatment of VOCs by plasma technology is that the organic waste gases can be partially degraded by plasma, such as the simple organic waste gases alkanes and Amines, which can be partially degraded But the excitation of organic pollutants or even air is not selective, and the degradation of organic waste gases is incomplete in just a few to one second Part of the gas will also produce carbon monoxide, ozone and nitrogen oxide pollution gases, resulting in a large

*Corresponding author's e-mail: 406778002@qq.com 
number of intermediate products, may be volatile, mobility, toxicity, greater harm.

1.2.3. The technology of photocatalysis waste gas treatment equipment is a special treatment method which uses special ultraviolet wave band, under the function of Special Catalyst Oxidizer, catalyzes oxygen to produce negative oxygen ion, and then oxidizes and reduces waste gas molecule. In fact, the degradation of organic waste gas by ultraviolet tubes used in the photocatalytic oxidation treatment equipment on the market is greatly influenced by catalytic materials, dust, reaction time, humidity, concentration of waste gas, etc. It will produce intermediate by-products and increase the pollution discharge. There are huge environmental liability risks.

1.2.4. Biodegradation of VOCs is mainly composed of microbial decomposable substances, and pollutants are the food source of microorganisms The pollutants that can be biotreated include: Organic compounds composed of Hydrocarbon, hydrogen and oxygen, Simple Organic Sulfides, organic nitrogen compounds, hydrogen sulfide and ammonia, etc. It requires small volume, low concentration, continuous exhaust, large capacity of exhaust gas treatment. Although the treatment process is more environmentally friendly, the operation and maintenance are complicated.

1.2.5. Combustion method is divided into direct combustion and catalytic combustion, the difference is whether to use catalyst to reduce the combustion temperature in the furnace. Because the heat storage combustion chamber temperature is generally not lower than 850 degrees, or even as high as 1000 degrees, it is inevitable to produce fuel nitrogen oxide. However, in the lower temperature of catalytic combustion, the chlorine ion in the water and the chlorine ion in the organic matter can easily form dioxins in the catalytic combustion chamber $\left(200 \sim 500{ }^{\circ} \mathrm{c}\right)$. The dioxin-producing temperature window of $\mathrm{msw}$ incineration is very short, and the high temperature of the back end above $800{ }^{\circ} \mathrm{C}$ can promote the dioxin decomposition, but the catalytic combustion process does not include the high temperature stage of the back end The dioxins produced during combustion are therefore released directly into the atmosphere.

\section{Comprehensive Treatment and emission reduction technology of VOCs in key industries}

\subsection{Comprehensive Treatment and emission reduction technology of VOCs in chemical industry}

We will focus on increasing the containment level of major VOCs-related processes, strengthening the collection of unorganized emissions, and increasing efforts to store and handle VOCs-containing materials.
Waste Water Storage, aeration tank and prior waste water treatment facilities shall be covered and sealed according to requirements, and waste gas collection and treatment shall be carried out. Actively promote the use of low VOCs content or low reactivity of raw and auxiliary materials, accelerate process improvement and product upgrading. Optimization of the production process, the pesticide industry to promote the water-phase, bio-enzyme synthesis technology; pharmaceutical industry to promote the use of bio-enzyme synthesis technology; rubber industry to promote the use of series mixing, atmospheric continuous desulfurization process. [2]

\subsection{Comprehensive Treatment and emission reduction technology of VOCs for industrial coating}

Strengthen source control, accelerate the use of powder, water-based, high-solid, radiation curing and other low content of VOCs coatings to replace solvent-based coatings ${ }^{[3]}$. The use of water-based paint in automobile manufacturing primer, high-solid or water-based paint in passenger car and color paint will be promoted in key areas, and the transformation of medium-and color paint on passenger cars and trucks will be accelerated. Water-based coatings are widely used in the process of making steel containers, such as inside, outside and wood floor painting. Wood furniture manufacturing promote the use of water-based, radiation curing, powder coatings and water-based adhesives; metal furniture manufacturing to promote the use of powder coatings; soft furniture manufacturing to promote the use of water-based adhesives. Construction Machinery Manufacturing vigorously promote the use of water-based, powder and high-solid coatings. The use of powder, water-based, radiation curing coatings and so on is promoted in the manufacture of electronic products.

\subsection{Comprehensive Treatment and emission reduction technology of VOCs in packaging and printing industry}

Efforts will be focused on the treatment of VOCs, including plastic flexible packaging and printing and iron cans. Efforts will be made to replace VOCs with raw and auxiliary materials with low (No) VOCs content and environment-friendly technologies. The major regions have gradually carried out the treatment of VOCs for publication printing, and promoted the use of vegetable oil-based inks, radiation-curable inks, low (no) content of raw and auxiliary materials such as VOCs and alcohol-based fountain solutions, as well as technologies such as waterless printing and automatic cleaning of blanket To reduce pollution and emissions. 


\section{The importance of strengthening environmental regulation}

\subsection{Regulation by the Environmental Protection Administration}

\subsubsection{Strengthening source list management}

To organize the updating investigation of VOCs emissions from key enterprises, focusing on the investigation of raw and auxiliary materials and products, main production processes, VOCs emission links, treatment measures and effects, VOCs emissions and VOCs inventory, find out the status of VOCs emission in enterprises, improve the list of emission sources, establish the database of VOCs major pollution sources $^{[4]}$.

\subsubsection{Strictly supervise and enforce the law}

We will continue to strengthen the monitoring of VOCs emissions, and conduct annual monitoring of key listed enterprises. The operation of VOCs treatment facilities and the purchase, replacement and disposal of consumables for treatment facilities are listed as the key points of on-site law enforcement to ensure the stable and effective operation of VOCs treatment facilities. To build an online monitoring system for VOCs treatment facilities on the Internet, and comprehensively strengthen the monitoring of the operation of major VOCs pollution collection and treatment facilities, on the management of abnormal use facilities, VOCs emissions can not meet the standards of enterprises should be investigated and dealt with.

\subsubsection{Play a leading role in science and technology}

Supporting various innovative bodies to carry out pilot demonstration of research and development and application of VOCs pollution control technologies, as well as the promotion and application of advanced and applicable technologies for VOCs emission; and encouraging enterprises to adopt a combination of independent research and development and overseas introduction, developing Technologies and equipment for the disposal of VOCs waste gases with Intellectual Property Rights; deepening cooperation among industries, universities and research institutes; and encouraging joint research on VOCs emission control countermeasures and technical policy, support the R \& D, Promotion and Application of water-based coatings, inks, adhesives and low toxicity, low harm and low Volatile organic compound content products, as well as the optimization of production process and process management in key industries.

\subsubsection{To step up publicity and education}

Giving full play to the role of the news media in the protection of the atmospheric environment, carrying out in-depth environmental publicity and education activities, popularizing knowledge of the protection of the atmospheric environment, and actively publicizing the importance, urgency and measures that can be taken to prevent and control VOCs pollution, we will raise public awareness of environmental protection and constantly enhance the ability of the public to participate in environmental protection ${ }^{[5]}$. Publicize advanced typical cases, guide and encourage the public and the media to carry out supervision by public opinion, and create a good social atmosphere for improving the quality of the atmospheric environment.

\subsection{The regulation of the enterprise itself}

Companies should incorporate the management and monitoring of VOCs into their daily production management systems, establish VOCs environmental management ledgers and update them annually Account Information should include: Enterprise basic situation, enterprise production situation, raw and auxiliary materials use account, VOCs treatment facilities operating account, etc. Enterprises included in the list of key regulatory enterprises, gradually in accordance with the national and provincial technical standards for on-line monitoring of VOCs, the industry by stages to promote the installation of on-line monitoring equipment VOCs terminal treatment devices. Mandatory disclosure of environmental information on pollution control for key enterprises, including on-line monitoring of enterprises, information on annual operation of pollution discharge and pollution control facilities, treatment efficiency, emission concentration, etc.

\section{Conclusion}

The current situation of VOCs emission and comprehensive treatment in key industries is severe, which needs the joint efforts of all sectors of society. The conclusions are as follows: First, To control VOCs, it is necessary to upgrade the industrial structure and promote the green transformation and upgrading of industry; Second, energy sector should optimized the energy structure to build a green and clean energy system; third, the department of transportation should adjusted the transport structure to speed up the development of Smart Green Transport; Fourth, the industrial enterprise should comprehensively deepen the control of industrial sources and strengthen the Coordinated Control of multiple pollutants; fifth, the department of ecology and environment should strengthen the control of mobile sources and deepen the coordinated prevention and control of pollution; sixth, the environmental erotection department should strengthen the comprehensive prevention and control of non-point sources and improve the level of fine management; seventh, various sectors of society should strengthen the joint prevention and control, responding effectively to polluted weather, and improved the ability to make scientific decisions on the prevention and control of air pollution. 


\section{Reference}

1. Volatile organic compound for key industries: Ministry of Ecology and Environment, 2019, Beijing.

2. Volatile Organic compound and emission reduction programme of Guangdong Province (2018-2020): Department of Ecological Environment of Guangdong Province, 2018, Guangzhou.

3. National Inventory of Advanced Pollution Control Technologies (field of air pollution control): Ministry of Ecological Environment, 2018, Beijing.

4. Draft for soliciting opinions on the method for calculating Volatile organic compound emissions from key industries in Guangdong Province (for trial implementation), 2019, Guangzhou.

5. Ye H(2019), Characteristics and control of Volatile organic compound pollution in typical industry, Chemical Industry Press, Beijing. 